nephron

Practice
Nephron 2017;136:287-291

DOI: $10.1159 / 000453531$
Received: April 12, 2016

Accepted after revision: November 15, 2016

Published online: December 16, 2016

\title{
An Overview of Errors and Flaws of Estimated GFR versus True GFR in Patients with Diabetes Mellitus
}

\author{
Sergio Luis-Lima ${ }^{a}$ Esteban Porrini ${ }^{b}$ \\ ${ }^{a}$ Department of Nephrology, University Hospital of the Canary Islands, Tenerife, and ${ }^{b}$ Centre for Biomedical \\ Research of the Canary Islands (CIBICAN), Instituto de Tecnologías Biomédicas (ITB), University of La Laguna, \\ Santa Cruz de Tenerife, Spain
}

\section{Key Words}

Estimated GFR - Type 2 diabetes mellitus · Glomerular filtration rate Formulas

\begin{abstract}
The determination of renal function is crucial in patients with type 2 diabetes (T2DM), a population at risk for chronic kidney disease (CKD). Glomerular filtration rate (GFR) can be measured (mGFR) with gold standard methods or estimated (eGFR) with formulas. Since 1957, when Effersoe published the first formula, more than 50 equations have been developed to estimate GFR. In this review, we examined the studies that compared mGFR and eGFR in patients with T2DM to analyze the performance of those formulae in this population. In cross-sectional studies, the average error of eGFR was $\pm 30 \%$ of mGFR. Thus, in a patient with mGFR of $60 \mathrm{~mL} /$ min, eGFR may vary from 42 to $78 \mathrm{~mL} / \mathrm{min}$. Moreover, many patients were misclassified according to CKD stages. Formulas failed to detect glomerular hyperfiltration. In longitudinal studies, eGFR poorly reflected real GFR decline over time. All studies showed that eGFR decline was slower than mGFR decline. Notably, no major improvement in accuracy and precision has been observed since 1957 despite the use of cystatin-c. Thus, formulas are not reliable indicators of GFR in patients with T2DM. In clinical studies, where GFR is the main outcome measure of the study, eGFR should be avoided.
\end{abstract}

(c) 2016 S. Karger AG, Basel (c) 2016 S. Karger AG, Basel

\section{KARGER}

E-Mail karger@karger.com

www.karger.com/nef
Type 2 diabetes (T2DM) affects $30-50 \%$ of patients with end-stage renal disease worldwide [1-3]. Unfortunately, this scenario has not changed in recent decades despite the increased use of renin-angiotensin system inhibitors. Thus, new strategies to prevent renal disease in T2DM patients are urgently needed. However, these strategies should consider a major problem in clinical research in nephrology: the lack of accuracy and precision of estimated glomerular filtration rate (eGFR) by formulae in reflecting true renal function. This is a crucial concern, since GFR and GFR decline are natural outcome measures in clinical studies. Moreover, the error of estimated renal function may jeopardize the possibility to detect potential benefits of new treatments. In this minireview, we will evaluate the evidence regarding the agreement between eGFR and measured GFR (mGFR) by gold standard methods in patients with T2DM.

\section{Cross-Sectional Studies}

Beauvieux et al. [4] evaluated a series of creatinine- or cystatin-c-based equations in 124 patients in whom GFR was measured with ${ }^{51} \mathrm{Cr}$-EDTA (Table 1). The proportion

Contribution from the CME course of the DIABESITY Working Group of the ERA-EDTA, Bergamo, December 4-5, 2015.

Dr. Esteban Porrini

Unidad de Ensayos Clínicos-UCICEC

University Hospital of the Canary Islands

Ofra s/n La Cuesta, ES-38320 La Laguna, Santa Cruz de Tenerife (Spain)

E-Mail esteban.l.porrini@gmail.com 


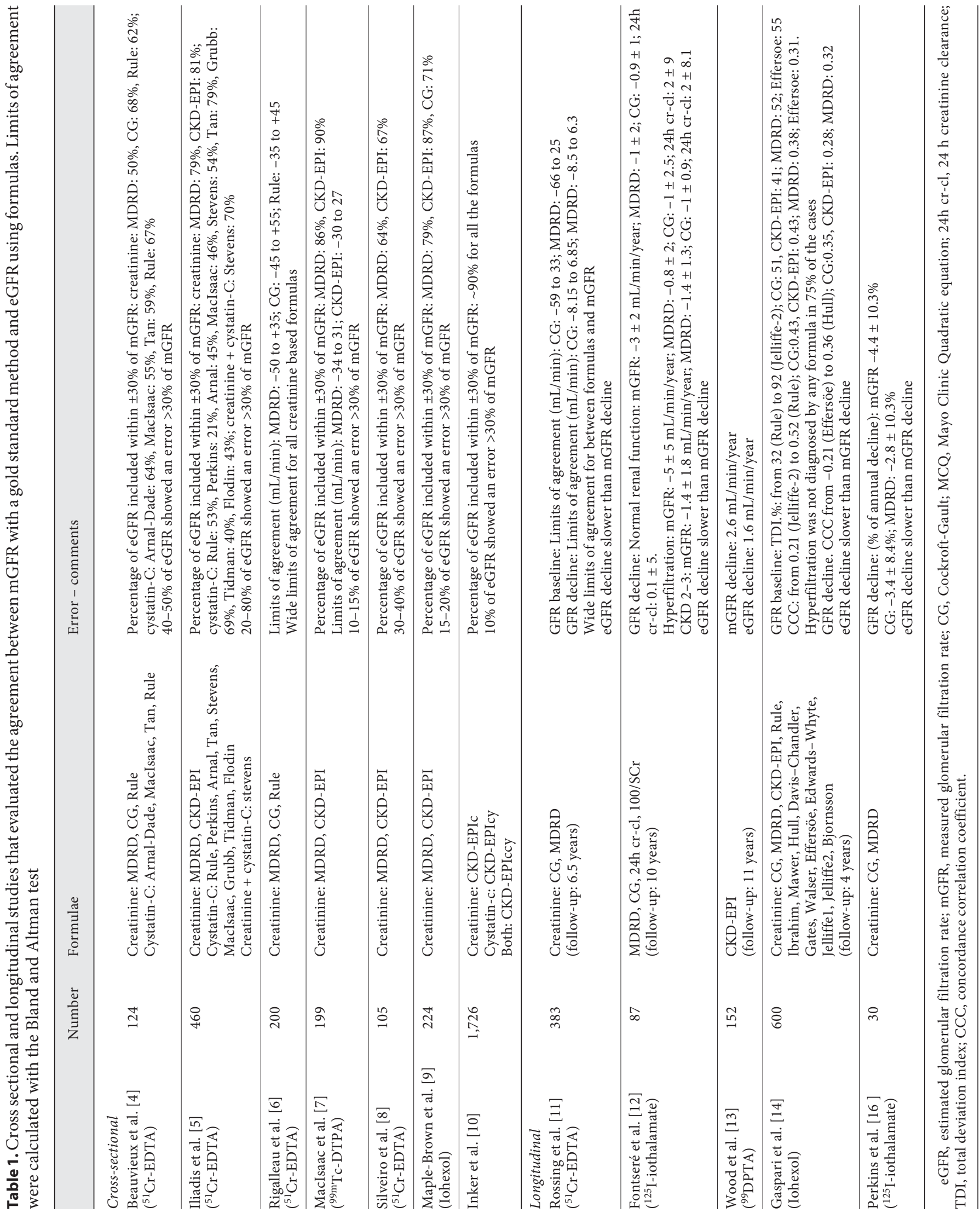


of eGFR values within $\pm 30 \%$ of mGFR ranged from $50 \%$ (Cockroft-Gault, CG) to 70\% (Rule and Modification of Diet in Renal Disease, MDRD; Table 1), indicating poor agreement between these formulae and mGFR. The boundary of $\pm 30 \%$ of $\mathrm{mGFR}$, which is a standard method to evaluate the performance of eGFR, is certainly a wide margin of error. For example, in a patient with $\mathrm{mGFR}$ of $60 \mathrm{~mL} / \mathrm{min}$, eGFR may range from 42 to $78 \mathrm{~mL} / \mathrm{min}$. Moreover, when $70 \%$ are included within this range, $30 \%$ of the estimations (one case in 3) have an even greater variability, that is, eGFR $<42$ and $>78 \mathrm{~mL} / \mathrm{min}$ for the above example. Such variability is clearly unacceptable from a clinical perspective.

Iliadis et al. [5] evaluated renal function in 460 patients with ${ }^{51} \mathrm{Cr}$-EDTA and 12 creatinine- or cystatin-c-based formulae. The proportion of eGFR values within $\pm 30 \%$ of mGFR ranged from 21\% (Perkins) to $81 \%$ (Chronic Kidney Disease Epidemiology Collaboration, CKD-EPI; Table 1). Rigalleau et al. [6] compared the CG, MDRD, and Rule equations with mGFR $\left({ }^{51} \mathrm{Cr}\right.$-EDTA $)$ in 200 patients. According to the Bland and Altman plot, formulae showed extreme limits of agreement with $\mathrm{mGFR}$, from -50 to $55 \mathrm{~mL} / \mathrm{min}$. As a consequence of this bias, $35 \%$ of the cases were misclassified based on chronic kidney disease (CKD) stages.

The lack of accuracy and precision of formulae have also been observed in patients with normal renal function. MacIsaac et al. [7] compared CKD-EPI and MDRD equations with mGFR (diethylenetriamine pentaacetic acid, DTPA) and observed wide limits of agreement, from -30 to $30 \mathrm{~mL} / \mathrm{min}$. Also, $80-90 \%$ of eGFR values fell within the wide limits of $\pm 30 \%$ of $\mathrm{mGFR}$. In the same line, Silveiro et al. [8] observed that both CKD-EPI and MDRD underestimated $\mathrm{mGFR}$ by about $20 \mathrm{~mL} / \mathrm{min}$.

In indigenous Australians, comparison between eGFR (CKD-EPI, CG, and MDRD) and mGFR (plasma clearance of iohexol) in a population at high risk for T2DM showed that $71-87 \%$ of the estimations fell within $\pm 30 \%$ of mGFR [9]. This error was similar in patients with and without impaired renal function.

Of note, this error was also evident in the original studies that described the formulas for the first time. Inker et al. [10] developed equations based on cystatin-c alone or in combination with creatinine in a subgroup of patients with diabetes. However, the P30 for the CKD-EPI equations with creatinine and or cystatin-c was similar, that is, 90\%.

Based on the above evidence, it seems clear that formula-derived estimations are grossly inaccurate in reflecting real renal function in patients with CKD or normal renal function. This wide error of $\pm 30 \%$ would be unacceptable for other measurements of risk factors like body mass in-

eGFR vs. True GFR in Patients with T2DM dex or blood pressure. Thus, standard thresholds of agreement used to validate a formula, that is, $\pm 30 \%$ of $\mathrm{mGFR}$ are too ample to be useful from a clinical point of view, since they lead to the acceptance of extreme variability between estimations and real renal function.

\section{Longitudinal Studies with Repeated Measurements of GFR}

One of the consequences of the errors in formulae is that they do not detect changes in GFR over time. Rossing et al. [11] evaluated 383 patients with T2DM and microalbuminuria or overt nephropathy. GFR was measured annually with ${ }^{51} \mathrm{Cr}$-EDTA and estimated CG and MDRD formulas during a mean follow-up of 6.5 years. At baseline, both formulas showed wide limits of agreement with mGFR, that is, from -66 to $31 \mathrm{~mL} / \mathrm{min}$. During follow-up, mean $\mathrm{mGFR}$ decline was $\sim 4-5 \mathrm{~mL} / \mathrm{min} /$ year while eGFR decline was $\sim 1 \mathrm{~mL} / \mathrm{min}$ slower. Also, eGFR decline showed wide limits of agreement compared with mGFR decline (Table 1). Fontseré et al. [12] evaluated 87 T2DM patients with normal renal function, glomerular hyperfiltration and CKD using mGFR (iothalamate) and eGFR with CG, MDRD, and 24-h creatinine clearance every 24 months during 10 years. As in the previous study, eGFR decline was slower than the real GFR decline. In general, GFR decline assessed by CG or MDRD only reflected 25\% of real decline, that is, $-4 \mathrm{~mL} / \mathrm{min} /$ year (mGFR) vs. -1 $\mathrm{mL} / \mathrm{min} /$ year (eGFR). In patients with CKD, mGFR and eGFR decline were similar, but the decrease in the number of patients $(n=13)$ limits the interpretation of this result. Finally, 24-h creatinine showed stable GFR decline or even improvement of renal function over time compared with mGFR decline. A slower renal function decline when evaluated with eGFR than mGFR in patients with T2DM has also been described for the CKD-EPI equation [13].

Gaspari et al. [14] evaluated the performance of 15 creatinine-based formulae in 600 patients with T2DM in whom GFR was measured by plasma clearance of iohexol (mGFR) every 6 months during a mean follow-up of 4 years. The authors evaluated the bias between eGFR and mGFR with specific statistics of agreement for continuous variables: the concordance correlation coefficient (CCC), the total deviation index (TDI), and the coverage probability (CP) [15]. In brief, CCC simultaneously combines accuracy and precision, and it is scored from 0 to 1 , and a value $>0.90$ reflects excellent concordance. TDI captures a large proportion of data within a boundary for

Nephron 2017;136:287-291

DOI: $10.1159 / 000453531$ 
Table 2. Clinical examples of the agreement between mGFR (iohexol plasma clearance) and eGFR estimated with the CKD-EPI group of formulas

\begin{tabular}{lllll}
\hline Case & $\begin{array}{l}\text { Measured GFR } \\
\text { (iohexol), mL/min }\end{array}$ & $\begin{array}{l}\text { CKD-EPI } \\
\text { (creatinine), } n(\%)\end{array}$ & $\begin{array}{l}\text { CKD-EPI } \\
\text { (cystatin-c), } n(\%)\end{array}$ & $\begin{array}{l}\text { CKD-EPI } \\
\text { (creatinine }+ \text { cystatin-c), } n(\%)\end{array}$ \\
\hline 1 & 20 & $14(-30)$ & $15(-25)$ & $12(-40)$ \\
2 & 29 & $61(110)$ & $71(145)$ & $56(93)$ \\
3 & 40 & $27(-33)$ & $32(-20)$ & $29(-28)$ \\
4 & 45 & $65(44)$ & $36(-20)$ & $41(-9)$ \\
5 & 64 & $53(-17)$ & $55(-14)$ & $44(-31)$ \\
6 & 70 & $84(20)$ & $74(6)$ & $67(-4)$ \\
7 & 90 & $86(-4)$ & $90(0)$ & $76(-16)$ \\
8 & 122 & $124(2)$ & $146(20)$ & $142(16)$ \\
9 & 150 & $139(-7)$ & $158(5)$ & $153(2)$ \\
\hline
\end{tabular}

allowed differences between estimations and measurements. This is considered the best approach to evaluate the agreement between eGFR and mGFR [15]. The results of this study are discouraging. At baseline, TDI for the 15 formulae was $\sim 40 \%$, which means that $90 \%$ of the estimations fell within $\pm 40 \%$ of mGFR. This was observed in patients with $\mathrm{CKD}$, normal renal function, or glomerular hyperfiltration. Also, these formulae failed to detect hyperfiltration in most cases. Renal function decline was slower when estimated with formulas than when measured with the gold standard, that is, mean mGFR decline $-3.37 \mathrm{~mL} / \mathrm{min} /$ year vs. eGFR decline which ranged from -1.34 to $0.34 \mathrm{~mL} / \mathrm{min} /$ year. Accordingly, eGFR showed low concordance with mGFR decline (CCC $<0.40$ for all the formulae). The first equation that was developed to estimate renal function (Effersoe 1957) showed a bias comparable to that of more recent formulae like MDRD, CKD-EPI, or even cystatin-based equations (Table 1). Thus, from a historical perspective, no improvement has been observed in the last 50 years in the estimation of GFR, despite the availability of more than 50 formulae and the use of cystatin-c. Finally, the error of eGFR was similar between the formulae that were adjusted or unadjusted for body surface area. The above studies consistently showed that eGFR decline is slower than mGFR decline, making these equations unacceptable for monitoring kidney function in patients with T2DM.

\section{Clinical Examples}

Evaluation of clinical cases is complementary to the analysis of the agreement between mGFR and eGFR. Table 2 shows the performance of 3 formulas of the CKDEPI "family": creatinine-, cystatin-, and creatinine-cys- tatin-based equations in patients with T2DM with diverse degrees of renal function, in whom GFR was measured by plasma clearance of iohexol at Hospital Universitario de Canarias (Tenerife, Spain). Of note, none of these patients had extreme obesity, anorexia, severe sarcopenia, cirrhosis, renal or liver transplantation, or other diseases that could influence creatinine or cystatin-c metabolism. All 3 formulas underestimated true GFR in patient 1 and overestimated true GFR in patient 2, despite a difference of only $9 \mathrm{~mL} /$ min between the 2 patients. In patients 1 and 5 , all the formulae underestimated the real GFR. On the other hand, these formulae showed one of the following: an acceptable error $(<10 \%)$, underestimation, or overestimation of GFR in the same patient (cases 3, 4, 6, and 7). For the patients with hyperfiltration (cases 8 and 9), all but one equation reflected the real GFR properly. These cases illustrate a major characteristic of the bias of eGFR, that is, the error is random, not systematic, and therefore unpredictable.

\section{Conclusions}

Patients with T2DM are at risk for major complications like CKD, cardiovascular events, blindness, peripheral neuropathy, and cancer. Many sophisticated methods have been developed to evaluate these diseases. However, renal function, a major outcome in this population is still estimated with an unreliable tool, namely, the eGFR. In patients with T2DM, the estimation of renal function using formulae, either creatinine- or cystatinbased, shows a wide margin of error, which averages $\pm 30 \%$ of real GFR, observed in several cross-sectional and longitudinal studies. This error leads to frequent misclassification of CKD, which limits the risk prediction for disease progression. Also, eGFR is not suitable to detect ear- 
ly stages of the disease (hyperfiltration) and monitor renal function over time. Importantly, no improvement in the accuracy and precision of eGFR has been observed in the last 5 decades. Use of eGFR in clinical research should be avoided whenever renal function is the main outcome measure of the study. New methods to assess renal function with sufficient accuracy and precision are urgently needed [17]. Finally, the comparison between eGFR and mGFR has to be performed with appropriate statistics of agreement, such as TDI, CCC, and CP, as proposed by Lin et al. [15] using restricted limits of agreement. The use of $\pm 30 \%$ of mGFR to indicate acceptable agreement between eGFR and mGFR represents a wide margin of error and should therefore be avoided.

\section{Acknowledgments}

S.L.L. is a research fellow supported by the Instituto de Salud Carlos III (Grants for Río Hortega specialized healthcare posttraining contracts), ISCIII CM15/00214, Spain. The authors grate- fully acknowledge support from (a) Instituto de Salud Carlos III for the following grant: PI13/00342, and Fondos FEDER for the following grants: PI 07/0732, the REDINREN RD/0021/0008, and PI10/02428. (b) Fundación Caja Canarias for the following grant: DIAB05. (c) We thank the IMBRAIN project for support (FP7-RE6-POT-2012-CT2012-31637-IMBRAIN) funded under the 7th Frameworks Programme capacities. DIABESITY working group of the ERA-EDTA.

\section{Search Strategy}

We searched PubMed for articles published in English with the terms "glomerular filtration rate," "estimated glomerular filtration rate," "measured glomerular filtration rate," "type 2 diabetes," "iohexol," "DTPA," "iothalamate," "Cr-EDTA," "glomerular hyperfiltration," "diabetic nephropathy," and "GFR decline." No date restrictions were placed on searches.

\section{Disclosure Statement}

The authors have no conflicts of interest to declare.

\section{References}

1 Global, regional, and national age-sex specific all-cause and cause-specific mortality for 240 causes of death, 1990-2013: a systematic analysis for the Global Burden of Disease Study 2013. Lancet 2015;385:117-171.

2 https://www.renalreg.org/reports/2014-seventeenth-annual-report/ (accessed April 4, 2015).

3 Schaubel DE, Morrison HI, Desmeules M, Parsons DA, Fenton SS: End-stage renal disease in Canada: prevalence projections to 2005. CMAJ 1999;160:1557-1563.

4 Beauvieux MC, Le Moigne F, Lasseur C, Raffaitin C, Perlemoine C, Barthe N, Chauveau $\mathrm{P}$, Combe C, Gin H, Rigalleau V: New predictive equations improve monitoring of kidney function in patients with diabetes. Diabetes Care 2007:30:1988-1994.

5 Iliadis F, Didangelos T, Ntemka A, Makedou A, Moralidis E, Gotzamani-Psarakou A, Kouloukourgiotou T, Grekas D: Glomerular filtration rate estimation in patients with type 2 diabetes: creatinine- or cystatin C-based equations? Diabetologia 2011;54:2987-2994.

6 Rigalleau V, Lasseur C, Raffaitin C, Perlemoine C, Barthe N, Chauveau P, Combe C, Gin $\mathrm{H}$ : The Mayo Clinic quadratic equation improves the prediction of glomerular filtration rate in diabetic subjects. Nephrol Dial Transplant 2007;22:813-818.

7 MacIsaac RJ, Ekinci EI, Premaratne E, Lu ZX, Seah JM, Li Y, Boston R, Ward GM, Jerums
G: The Chronic Kidney Disease-Epidemiology Collaboration (CKD-EPI) equation does not improve the underestimation of Glomerular Filtration Rate (GFR) in people with diabetes and preserved renal function. BMC Nephrol 2015;16:198.

8 Silveiro SP, Araújo GN, Ferreira MN, Souza FD, Yamaguchi HM, Camargo EG: Chronic Kidney Disease Epidemiology Collaboration (CKD-EPI) equation pronouncedly underestimates glomerular filtration rate in type $2 \mathrm{di}$ abetes. Diabetes Care 2011;34:2353-2355.

9 Maple-Brown LJ, Ekinci EI, Hughes JT, Chatfield M, Lawton PD, Jones GR, Ellis AG, Sinha A, Cass A, Hoy WE, O’Dea K, Jerums G, MacIsaac RJ; investigators of the eGFR Study: Performance of formulas for estimating glomerular filtration rate in Indigenous Australians with and without Type 2 diabetes: the eGFR Study. Diabet Med 2014;31:829-838.

10 Inker LA, Schmid CH, Tighiouart H, Eckfeldt JH, Feldman HI, Greene T, et al: Estimating glomerular filtration rate from serum creatinine and cystatin C. N Engl J Med 2012;367: 20-29.

11 Rossing P, Rossing K, Gaede P, Pedersen O, Parving HH: Monitoring kidney function in type 2 diabetic patients with incipient and overt diabetic nephropathy. Diabetes Care 2006;29:1024-1030.

12 Fontseré N, Salinas I, Bonal J, Bayés B, Riba J, Torres F, Rios J, Sanmartí A, Romero R: Are prediction equations for glomerular filtration rate useful for the long-term monitoring of type 2 diabetic patients? Nephrol Dial Transplant 2006;21:2152-2158.

13 Wood AJ, Churilov L, Perera N, Thomas D, Poon A, MacIsaac RJ, Jerums G, Ekinci EI: Estimating glomerular filtration rate: performance of the CKD-EPI equation over time in patients with type 2 diabetes. J Diabetes Complications 2016;30:49-54.

14 Gaspari F, Ruggenenti P, Porrini E, Motterlin N, Cannata A, Carrara F, Jiménez Sosa A, Cella C, Ferrari S, Stucchi N, Parvanova A, Iliev I, Trevisan R, Bossi A, Zaletel J, Remuzzi G; GFR Study Investigators: The GFR and GFR decline cannot be accurately estimated in type 2 diabetics. Kidney Int 2013;84:164-173.

15 Lin L, Hedayat AS, Wu W: Statistical Tools for Measuring Agreement, ed 1. New York, Springer, 2012.

16 Perkins BA, Nelson RG, Ostrander BE, Blouch KL, Krolewski AS, Myers BD, Warram JH: Detection of renal function decline in patients with diabetes and normal or elevated GFR by serial measurements of serum cystatin $\mathrm{C}$ concentration: results of a 4-year follow-up study. J Am Soc Nephrol 2005;16: 1404-1412.

17 http://www.metabolon.com/news-2016/metabolon-to-develop-accugfr-kidney-function-test-with-johns-hopkins-and-tuftsmedical-center.aspx. 